\title{
INTESTINAL FLORA AFFECTS GUT-BRAIN AXIS
}

\section{F. Orzi}

\section{La Sapienza University, Rome, Italy}

\begin{abstract}
Extreme changes of the gut microbiota affect brain function and behaviour, as measured in animal models. A question arises as to the potential clinical relevance of these findings, especially when one considers the influence of dietary habits on the gut flora diversity. The issue attracts public awareness and media attention, causing perhaps diffusion of more interpretations than solid data. A growing body of knowledge, however, supports a role for different gut bacteria, or dietary antigens, in affecting the immune system function, causing potential changes in clinically relevant susceptibility to noxious stimuli.
\end{abstract}

Key words: gut, microbiota, gut-brain axis, limbic system, stress, immune response

An organism function relies on the interaction among the different organs, which compose the organism. While this is a self-evident naïve statement, a difficulty arises when one comes to define and measure the interactions. The gut-brain axis is among the most documented interaction systems. It also bears cultural and philosophical issues, which attract public awareness through the media, in terms of the health benefits of probiotics or potential effects of our gut guests on the most recondite function of the brain, the mind. Links between gastrointestinal pathology and psychiatric or neurological disorders have been described for decades and supported by pathology and epidemiology data. For instance, there is evidence for spreading of the Parkinson's pathology trans-synaptically from the enteric nervous system, via the vagus nerve, to the brain, ${ }^{1}$ probably maintained by environmental factors. ${ }^{2}$ Gastro enteric and psychiatric co-morbidity has been well known for decades. Findings prove a two-way connection gutbrain. Causality, however, often remains a question, as establishing whether a phenomenon is the cause or consequence of the other remains an elusive task.

A great deal of data uncovers the effect of the gut on relatively simple behaviors, such as the feeding behavior in experimental animals. Individuals as "simple" as the $C$. elegans nematode show appetite control. They mimic the behavioral sequence of satiety and postprandial sleep in rodents and humans, while starvation induces food seeking behavior. Neuro-humoral mechanisms signal to the nervous system the status of the fat storage and cause the appropriate behavioral response. Basic mechanisms are common to nematodes as well humans, and the consistent similarity expresses the efficacy of the homeostatic mechanism for survival. Major fat-regulatory pathways, conserved between the C. elegans and the mammals, include insulin-like, serotonin and leptin.

In rodents and mammals, the basic homeostatic mechanisms also present in the C. elegans, are integrated with reward-related inputs. The integration can be studied in laboratory, during self-administration settings. The motivational properties of food and the function of the reward pathways can be, therefore, measured under different conditions, including starvation or satiety. For instance, weight loss lowers plasma levels of insulin and leptin and increases ghrelin levels. The changes are associated with enhancement of the rewarding properties of food, as shown by changes in the motivational behavior. And the behavioral changes express biochemical effects and functional activation of selected brain areas within the reward system. Conversely, a positive energy balance, decreases the rewarding properties of food and reduces the likelihood of activation of the reword brain areas. Gut hormones, in addiction, affect synaptic plasticity and learning mechanisms. From an evolutionary perspective, it is not surprising that gut signals have an influence on memory and cognition. ${ }^{3}$ 
In the last 15 years evidence has grown regarding a role of gut microbiota. Early studies referred to the effects of microbiota in regulating energy storage. In line with previous studies on the gut-brain axis, a number of authors have shown that the microbiota integrates the mechanisms involved in feeding behavior. For instance, germ-free rodents exhibit reduced body fat content, reduced oxygen metabolism and increased chow consumption, with respect to control. ${ }^{4}$

A work by Sudo et al. ${ }^{5}$ was a pioneer one in showing effects of the microbiota extending beyond the boundaries of the feeding behavior. The authors reported that germ-free rodents show altered response to the hypothalamic-pituitary-adrenal (HPA) axis to the stress. Because the HPA response to stress is mediated by the limbic system, the findings proved for the first time an effect of the microbiota on cognitive processing.

Data have accumulated since then on the effects of gut microbiota on the brain. ${ }^{6}$ Most of the data are being obtained in extreme conditions, such as germ-free animals or intensive antibiotic treatments. Caution, therefore, needs to be used when trying to extend the experimental data to clinical conditions. However, the experimental data are solid and they strongly suggest (but in most cases do not prove) that manipulation of the human microbiota has therapeutic effects for several diseases or conditions. ${ }^{7}$

Recent data have focused on effect of microbiota on the immune response. It is worth reminding that one of the main immune precursor effectors, the $\mathrm{CD} 4^{+} \mathrm{T}$ cell, differentiates to numerous subsets, including THI7 and regulatory T cells (Treg cells). Each subtype cell is specialized to combat certain types of infection or resolve inflammation. TH17 cells promote the inflammatory process. Treg contribute to prevention of the immune response by producing IL-10, which suppresses the aberrant activation of THI 17 and $\gamma \delta$-T cells.

Consistent data show that different gut bacteria, or dietary antigens, drive the $\mathrm{CD} 4^{+} \mathrm{T}$ differentiation toward a pro- or anti-inflammatory response. ${ }^{8}$ As a straightforward consequence, the gut microbiota needs to represent the appropriate variety of different bacteria to favorite balanced immune response.

Following a large wealth of data approaching the relevance of the immune response in the susceptibility of the nervous system to the ischemic stroke, ${ }^{9}$ a remarkable paper by Iadecola's group at the Cornell University reports the effect of the treatment for 2 weeks with the antibiotics amoxicillin and clavulanic acid on the infarct size, in a mouse model of stroke. ${ }^{10}$ The treatment reduces the cerebral infarct size following occlusion of the middle cerebral artery. The effect is consistent with a reduced inflammatory response to the ischemic insult. The treated animals did in fact show a reduced frequency of intestinal effector IL- $17^{+} \gamma \delta$-T cell. The reduction was observed also in the leptomeninges, revealing a directed trafficking of the effector $\mathrm{T}$ cells from the gut to the lep- tomeninges. The reduction was associated with reduced IL-17-responsive chemokine expression in the brain parenchyma. The beneficial effect of the antibiotic treatment is, therefore, to be interpreted as the result of a protective reduction of the IL-17 mediated post-ischemic inflammation. IL-17 and $\gamma \delta$-T cells have been implicated in human stroke as well. ${ }^{11}$

A question, obviously, concerns the potential clinical relevance of these findings when one considers the influence of dietary habits on the gut flora diversity. An ongoing debate regards the claimed deleterious effects of high salt dietary intake and whether there is evidence for restricting sodium intake in the general population. ${ }^{12,13}$ In mice, chronic high dietary salt causes changes in cerebral blood flow and cognitive impairment. The effect depends on expansion of TH17 cells in the small intestine, resulting in marked increase in plasma IL-17. ${ }^{14}$ According to a recent work, ${ }^{15}$ in the mouse, high salt diet affects the gut flora, particularly by depleting Lactobacillus murinus. The authors observed that the salt increases the blood pressure together with the frequency of intestinal TH17 cells. Treatment with Lactobacillus murinus remarkably reverts the effects.

The data, altogether, strongly suggest an influence of dietary habits on the immune system function, causing potential changes in clinically relevant susceptibility to noxious stimuli.

\section{REFERENCES}

1. Klingelhoefer L, Reichmann H. Pathogenesis of Parkinson disease: the gut-brain axis and environmental factors. Nat Rev Neurol 2015;11:625-36.

2. Reichmann H. View point: Etiology in Parkinson's disease. Dual hit or spreading intoxication. J Neurol Sci 2011;310:9-11.

3. Gómez-Pinilla F. Brain foods: the effects of nutrients on brain function. Nat Rev Neurosci 2008;9:568-78.

4. Bäckhed F, Ding H. Wang T, et al. The gut microbiota as an environmental factor that regulates fat storage. Proc Natl Acad Sci USA 2004;101:15718-23.

5. Sudo N, Chida Y, Aiba Y, et al. Postnatal microbial colonization programs the hypothalamic-pituitary-adrenal system for stress response in mice. $J$ Physiol 2004;558:263-75.

6. Bailey MT, Cryan JF. The microbiome as a key regulator of brain, behavior and immunity: Commentary on the 2017 named series. Brain Behav Immun 2017;66:18-22.

7. Lynch SV, Pedersen O. The Human Intestinal Microbiome in Health and Disease. N Engl J Med 2016;375:2369-79.

8. Honda $K$, Littman DR. The microbiota in adaptive immune homeostasis and disease. Nature 2016;535:75-84.

9. Garcia-Bonilla L, Benakis C, Moore J, et al. Immune mechanisms in cerebral ischemic tolerance. Front Neurosci 2014;8:44.

10. Benakis C, Brea D, Caballero S, et al. Commensal microbiota affects ischemic stroke outcome by regulating intestinal $\gamma \delta T$ cells. Nat Med 2016;22:516-23.

11. Gelderblom $M$, Leypoldt $F$, Steinbach $K$, et al. Temporal and spatial dynamics of cerebral immune cell accumulation in stroke. Stroke 2009;40:1849-57. 
12. Nicholls MG. Population-wide dietary sodium restriction: a cautious view. Curr Hypertens Rep 2011;13:325-7.

13. Kotchen TA, Cowley AW, Frohlich ED. Salt in Health and Disease: A Delicate Balance. N Engl J Med 2013;368:2531-2.

14. Faraco G, Brea D, Garcia-Bonilla L, et al. Dietary salt promotes neurovascular and cognitive dysfunction through a gut-initiated TH17 response. Nat Neurosci 2018;21:240-9.

15. Wilck N, Matus MG, Kearney SM, et al. Salt-responsive gut commensal modulates TH17 axis and disease. Nature 2017;551: 585-9. 